\section{Spontaneous regression of pulmonary pure \\ ground-glass opacity with progression of a solid compo- nent during a 40-month follow-up}

\section{Nobuaki Ochi, ${ }^{1}$ Nagio Takigawa, ${ }^{1}$ Masayuki Yasugi, ${ }^{1}$ Daijiro Harada, ${ }^{2}$ Hiromichi Yamane, ${ }^{1}$ Mitsune Tanimoto, ${ }^{2}$ Katsuyuki Kiura ${ }^{2}$ \\ 'Department of General Internal Medicine 4, Kawasaki Hospital, Kawasaki Medical School; 2Department of Respiratory Medicine, Okayama University Hospital, Japan}

\section{Abstract}

A 75-year-old man with a 50 pack-year smoking history underwent a right upper lobectomy due to an early stage lung adenocarcinoma. Simultaneously, pure ground-glass opacity (GG0) on the left upper lobe measuring 6.7 $\mathrm{mm}$ in diameter was detected on computed tomography (CT), which was considered atypical adenomatous hyperplasia, a bronchioloalveolar carcinoma, or focal organizing pneumonia/fibrosis. Eighteen months later, the diameter of the lesion increased to $9.0 \mathrm{~mm}$. The lesion further enlarged to $10.4 \mathrm{~mm}$ with a small solid component within the GG0 at 28 months after the initial CT scan. At the 33month follow-up, the lesion had decreased in size and a solid component was prominent. Forty months after the initial CT, the lesion seemed to be a fibrotic scar. To the best of our knowledge, no studies have reported a pure GG0 progressing with a solid component that regressed spontaneously over such a long period. Although this case seems rare, physicians should be aware that a lung nodule compatible with progression from in situ carcinoma to invasive carcinoma on CT could resolve over 24 months.

\section{Introduction}

A ground-glass opacity (GG0) on chest computed tomography (CT) that increases in size is closely associated with atypical adenomatous hyperplasia (AAH) and bronchioloalveolar carcinoma (BAC). ${ }^{1}$ Small-sized GGOs generally grow slowly and subsequently have a solid component which is an indication for surgical resection ${ }^{2,3}$ We experienced a pure GG0 that enlarged with a solid component and became scarred spontaneously during a 40-month follow-up.

\section{Case Report}

A 75-year-old man with a 50 pack-year smoking history was referred for a lung adenocarcinoma (29 $\mathrm{mm}$ in diameter) in the right upper lobe. Simultaneously, a pure $\mathrm{GGO}$ was found on the left upper lobe by CT, measuring $6.7 \mathrm{~mm}$ in diameter (Figure 1A), which was suspected to be AAH, BAC, or focal organizing pneumonia/fibrosis. He had no symptoms and underwent a right upper lobectomy and mediastinal lymph node dissection (pT1bN0M0). Although the lesion in the left upper lobe did not change at the 6-month and the 12-month follow-up (Figure 1B), it had increased in size to $9.0 \mathrm{~mm}$ in diameter (Figure 1C) at 18 months after the initial CT scan. During the next 10 months, the lesion further enlarged to $10.4 \mathrm{~mm}$ in diameter (Figure 1D), with a small solid component within the GGO (Figure 2A). Because the lesion could progress from AAH/BAC to an invasive adenocarcinoma, we recommended a surgical resection. However, the patient pre-
Correspondence: Nagio Takigawa, Department of General Internal Medicine 4, Kawasaki Medical School, 2-1-80 Nakasange, Kita-ku, Okayama, 700-8505, Japan. Tel: +81-86-225-2111; Fax: +8186-232-8343. E-mail: ntakigaw@gmail.com

Key words: ground-glass opacity, atypical adenomatous hyperplasia, bronchioloalveolar carcinoma, non-small cell lung cancer, computed tomography.

Conflict of interest: the authors report no conflicts of interest.

Received for publication: 4 August 2011. Accepted for publication: 5 October 2011.

This work is licensed under a Creative Commons Attribution NonCommercial 3.0 License (CC BYNC 3.0).

\section{(C)Copyright N. Ochi et al., 2011}

Licensee PAGEPress, Italy

Chest Disease Reports 2011; 1:e14

doi:10.4081/cdr.2011.e14

ferred a careful follow-up on CT. An abnormal $\left[{ }^{18} \mathrm{~F}\right]$-fluorodeoxyglucose (FDG) uptake was
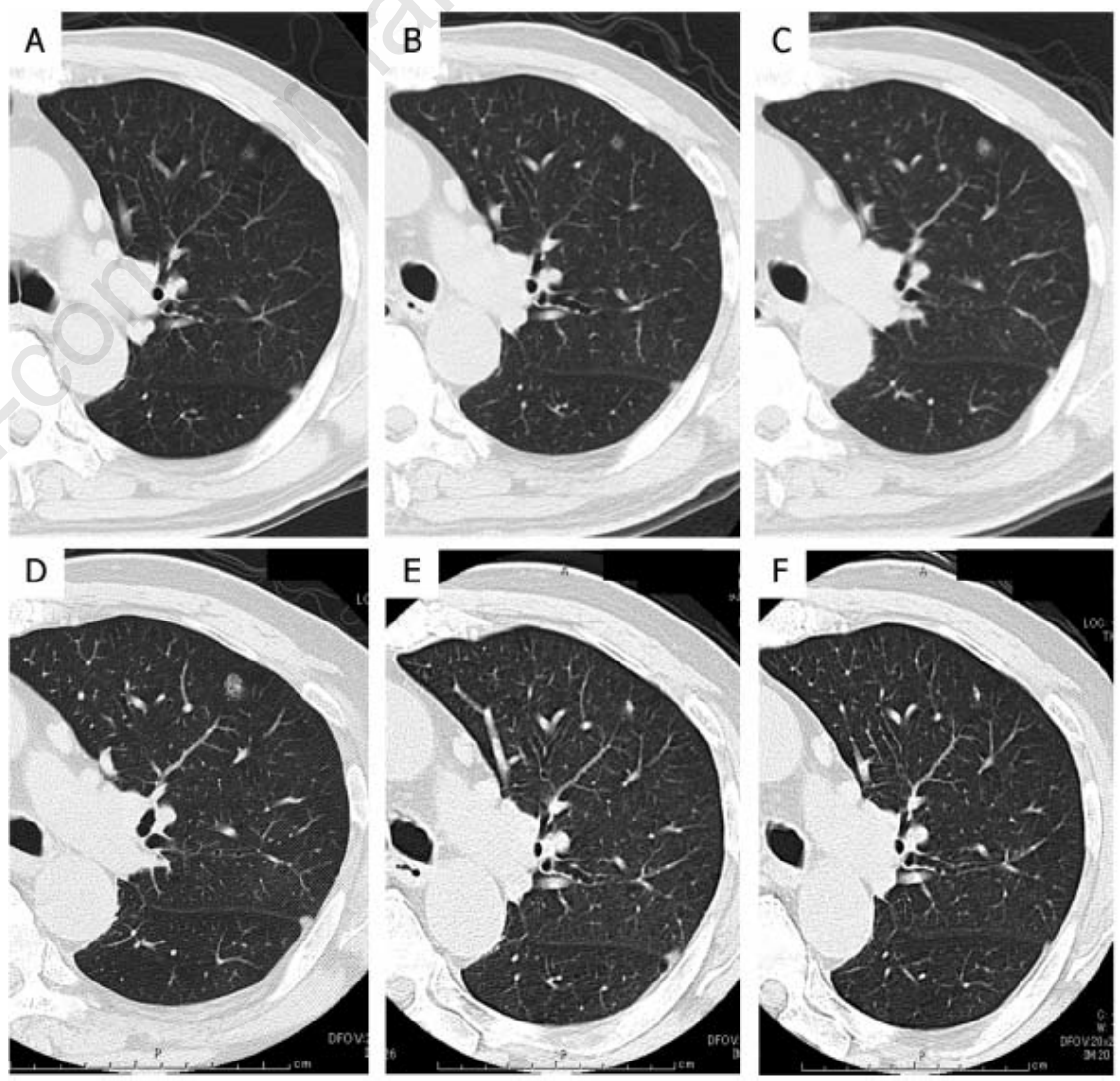

Figure 1. Computed tomography reveals focal pure ground-glass opacity on the left upper lobe of the lung. Images were obtained at the initial diagnosis (A), 12 months (B), 18 months (C), 28 months (D), 33 months (E), and 40 months (F) after the initial computed tomography scan. 
not detected in the lesion on positron emission tomography (PET)/CT after his initial CT scan and at 28-month follow-up. In addition, because the small and faint lesion could not be detected under radiographic guidance, the bronchoscopic approach was suspended. At the 33-month follow-up, the lesion had decreased in size to $4.9 \mathrm{~mm}$ (Figure 1E), and a solid component was prominent (Figure 2B). At the 40month follow-up, the lesion seemed to be a fibrotic scar (Figure 1F).

\section{Discussion}

Following an increase in a pure GG0, a solid part appeared in the center (mixed GG0), which was interpreted as progression from an in situ peripheral adenocarcinoma to invasive adenocarcinoma. ${ }^{4}$ The guidelines for managing small pulmonary nodules detected on CT include optimal follow-up intervals based on patient risks (e.g., smoking history, other risk factors) and size, but do not indicate when a biopsy or resection should be performed. ${ }^{5}$ The newer guideline for small pulmonary nodules is as follows. ${ }^{6}$ Conservative management of nodules between 5 and $10 \mathrm{~mm}$ in size with pure GG0 requires at least an initial follow-up examination in 3-6 months to document that lesions have not resolved spontaneously (or following antibiotic therapy). Similar to solitary lesions $10 \mathrm{~mm}$ or larger in size with GG0, any lesion with mixed solid component and GG0, regardless of size, represents malignancy with sufficient likelihood to warrant further evaluation. The evaluation should include the timely performance of PET or preferably PET/CT. At this time, we follow patients referring to the recommendation. If an internal solid component appears, such as in our case, physicians usually recommend surgical resection of the lesion. Because our patient was unwilling to undergo resection, we were able to observe the lesion.

This case had a limitation because no pathological diagnosis was made. We just focused on the long-term morphological change of the GG0 lesion. The possible differential diagnoses of the pure GGO are as follows: BAC, AAH, nonspecific fibrosis and focal organizing pneumonia. ${ }^{7}$ Among them, focal organizing pneumonia possibly progresses and regresses spontaneously during the observation. The inflammatory cells might spread and unspread around the focus very slowly although the exact mechanisms remain

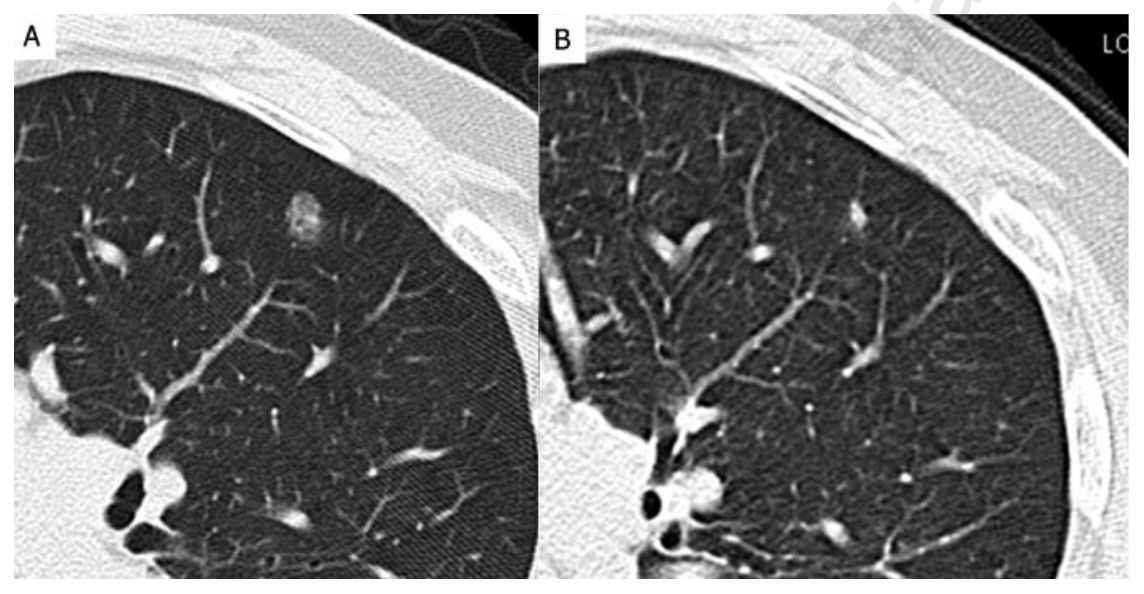

Figure 2. Magnification of the computed tomography images at 28 months (A) and at 33 months (B) after the initial computed tomography scan. unclear. A progression of a solid component in the GG0 might be the microscopic fibrotic change in the center of the GGO lesion. Because the GGO lesion finally decreased in size during the long time follow-up, it was regarded as an inflammatory lesion. After the GG0 lesion regressed, a solid component of fibrosis might remain. Although this case seems rare, physicians should know that lung nodules compatible with progression from AAH/BAC to invasive carcinoma on CT could resolve over 24 months.

\section{References}

1. Miller RR. Bronchioloalveolar cell adenomas. Am J Surg Pathol 1990;14:904-12.

2. Swensen SJ, Jett JR, Hartman TE, et al. Lung cancer screening with CT: Mayo Clinic experience. Radiology 2003;226: 756-61.

3. Nakata M, Saeki H, Takata I, et al. Focal ground-glass opacity detected by low-dose helical CT. Chest 2002;121:1464-7.

4. Travis WD, Garg K, Franklin WA, et al. Evolving concepts in the pathology and computed tomography imaging of lung adenocarcinoma and bronchioloalveolar carcinoma. J Clin Oncol 2005;23:3279-87.

5. MacMahon H, Austin JH, Gamsu G, et al. Guidelines for management of small pulmonary nodules detected on CT scans: a statement from the Fleischner Society. Radiology 2005;237:395-400.

6. Godoy MC, Naidich DP. Subsolid pulmonary nodules and the spectrum of peripheral adenocarcinomas of the lung: recommended interim guidelines for assessment and management. Radiology 2009;253:606-22.

7. Kim HY, Shim YM, Lee KS, et al. Persistent pulmonary nodular ground-glass opacity at thin-section CT: histopathologic comparisons. Radiology 2007;245:267-75. 